\title{
Simultaneous T-dualization of type II pure spinor superstring
}

\author{
B. Nikolića ${ }^{\text {, B. Sazdović }}{ }^{\mathrm{b}}$ \\ Institute of Physics Belgrade, University of Belgrade, Pregrevica 118, Belgrade, Serbia
}

Received: 31 October 2018 / Accepted: 10 December 2018 / Published online: 27 December 2018

(C) The Author(s) 2018

\begin{abstract}
In this article we consider simultaneous Tdualization of the type II superstring action in a pure spinor formulation. Simultaneous T-dualization means that we make T-dualization at the same time along some subset of initial coordinates denoted by $x^{a}$. The only imposed assumption stems from the applicability of the Buscher T-dualization procedure-background fields do not depend on the dualized directions $x^{a}$. In this way we obtain the full form of the T-dual background fields and T-dual transformation laws. Because the two chiral sectors transform differently, there are two sets of vielbeins and gamma matrices connected by a local Lorentz transformation. Its spinorial representation is the same as in the constant background case. We also found the full expression for the T-dual dilaton field.
\end{abstract}

\section{Introduction}

The importance of T-duality rose after M-theory was discovered. Five consistent superstring theories are connected by a web of $\mathrm{T}$ and $\mathrm{S}$ dualities and lead to M-theory [1-9]. For example, T-duality connects type IIA and type IIB superstring theories in the sense that after an odd number of $\mathrm{T}$ dualizations type IIA/B turns into IIB/A, while after an even number of T-dualizations type IIA/B stays unchanged [1013].

T-dualization of type II superstrings was a subject of Refs. [13-16]. In some articles T-dualization along a single direction is considered $[15,16]$. Two chirality sectors transform under T-duality differently and, consequently, in the T-dual picture there are two sets of vielbeins and gamma matrices. But there is a local Lorentz transformation connecting them. In Refs. $[15,16]$, in the case of T-dualization along

This work was supported in part by the Serbian Ministry of Education, Science and Technological Development, under contract no. 171031.

\footnotetext{
a e-mail: bnikolic@ipb.ac.rs

be-mail: sazdovic@ipb.ac.rs
}

one direction, a spinorial representation of that local Lorentz transformation is found. A detailed derivation is presented in Ref. [15].

The case of simultaneous T-dualization of pure spinor type II superstring theory is investigated in Ref. [13]. By simultaneous T-dualization we mean T-dualization along some subset of coordinates at the same time [13,17]. In Ref. [13] we used the action in the approximation of constant background fields obtained from the general one given in Ref. [18] after some assumptions. First, we took all background fields to be $x^{\mu}$ independent, justifying such an assumption with the possibility of making simultaneous T-dualization along any subset of coordinates including full T-dualization. The second crucial assumption was a technical one. Because the full action of Ref. [18] is in the form of an expansion in powers of $\theta^{\alpha}$ and $\bar{\theta}^{\alpha}$, for technical simplicity, we took into consideration only basic terms which are $\theta^{\alpha}$ and $\bar{\theta}^{\alpha}$ independent. Effectively, only physical superfields (their first components are identified with supergravity fields) survive and they are constant. Using the obtained action, in Ref. [13] we investigated simultaneous T-dualization and obtained the transformation laws connecting initial and T-dual coordinates and the expressions for T-dual background fields. We presented a detailed derivation of the local Lorentz transformation in the spinorial representation. Also we discussed the case of timelike T-dualization and prove the results of Ref. [19] obtained in the analysis of an effective action.

The mathematical framework for T-dualization was developed by Buscher [20,21]. The standard Buscher T-dualization procedure is applicable if the theory has a shift symmetry. This means that it is possible to find a coordinate basis such that the background fields do not depend on some directions [20-27]. Localization of the symmetry is done in a standard way-by replacing the world-sheet derivatives $\partial_{ \pm} x^{\mu}$ with covariant ones, $D_{ \pm} x^{\mu}=\partial_{ \pm} x^{\mu}+v_{ \pm}^{\mu}$, where $v_{ \pm}^{\mu}$ are gauge fields. In order to make the T-dual theory physically equivalent to the initial one, a term with Lagrange multiplier is added to the action so that the field strength should be zero. 
As a consequence of the shift symmetry, we can fix the initial coordinates and obtain the so-called gauge fixed action. Variation of this action with respect to the Lagrange multiplier produces the initial action, while variation with respect to the gauge fields gives the T-dual action. When one applies the procedure in the open string case [28,29], then one has to consider both the equations of motion and the boundary conditions. Consequently, $D p$-branes appear in the analysis.

There are two main approaches in superstring theory-the Neveu-Schwarz-Ramond (NSR) and the Green-Schwarz (GS) formalism [22-24]. The former contains explicit worldsheet supersymmetry, while the latter has explicit spacetime supersymmetry. There are some disadvantages of these approaches: in the NSR formalism the Ramond-Ramond (R$\mathrm{R})$ sector is missing and spacetime supersymmetry is not manifest, while in the GS formalism quantization can be performed just in light-cone gauge. In the last two decades a new approach has appeared, the pure spinor formalism $[18,30-39]$. It is pretty similar to the GS approach in the sense that spacetime supersymmetry is manifest but it contains pure spinors $\lambda^{\alpha}$ and $\bar{\lambda}^{\alpha}$ satisfying the so-called pure spinor constraints, $\lambda^{\alpha}\left(\Gamma^{\mu}\right)_{\alpha \beta} \lambda^{\beta}=\bar{\lambda}^{\alpha}\left(\Gamma^{\mu}\right)_{\alpha \beta} \bar{\lambda}^{\beta}=0$. The pure spinor formalism uses the advantages of the previous two formalisms and avoids some disadvantages. In this article we will use the pure spinor action of type II superstring from Ref. [18], where a detailed derivation of the action is presented. The action is given in the form of an expansion in powers of $\theta^{\alpha}$ and $\bar{\theta}^{\alpha}$ obtained using (anti)holomorphicity and nilpotency conditions.

In this article we study simultaneous T-dualization of the pure spinor superstring type II theory with only one assumption-background fields are independent of the coordinates $x^{a}$ along which we make T-dualization. This assumption stems from the applicability of the Buscher procedure. Our main goal is to find the full form of all T-dual background fields and T-dual transformation laws.

We start with the action (2.1) and decompose the variables $X^{M}$ and $\bar{X}^{M}$ (3.1) extracting directions $x^{a}$ along which we make T-dualization. Then we perform a Buscher Tdualization procedure along $x^{a}$ obtaining the T-dual transformation laws and T-dual action. The two chirality sectors transform differently under T-dualization. Consequently, there are two sets of vielbeins and gamma matrices, which are connected by the local Lorentz transformation represented by the matrix ${ }_{a} \Omega$. In order to work with a unique set of gamma matrices, we introduce proper fermionic variables keeping unbar fermionic variables unchanged, while bar variables are corrected by the matrix ${ }_{a} \Omega$. After introducing proper fermionic variables, we read the full form of the T-dual background fields.

We get the explicit expressions for T-dual physical superfields. For constant background they turn into the result of Ref. [13]. The expressions for the auxiliary superfields and field strengths are completely new in the sense that they are missed in the constant background case. In order to avoid long expressions, we will give explicitly just the expression for the T-dual auxiliary field ${ }_{a} A_{\alpha}^{a},(5.25)$, and the expression for the field strength ${ }_{a} \Omega^{a, \hat{\mu} \hat{v}}$, (5.26).

The dilaton field is treated within the quantum formalism. We obtain the most general expression for the T-dual dilaton field within a pure spinor formulation of type II superstring theory.

\section{Type II pure spinor superstring theory}

In this section we will introduce the type II pure spinor superstring action in compact and expanded form.

The sigma model action for type II superstring of Ref. [18] is of the form

$S=\int_{\Sigma} \mathrm{d}^{2} \xi\left(X^{T}\right)^{M} A_{M N} \bar{X}^{N}+S_{\lambda}+S_{\bar{\lambda}}$,

where $X^{M}$ and $\bar{X}^{N}$ are left and right chiral supersymmetric variables,

$$
\begin{gathered}
X^{M=}\left(\begin{array}{c}
\partial_{+} \theta^{\alpha} \\
\Pi^{\mu} \\
d_{\alpha} \\
\frac{1}{2} N^{\mu \nu}
\end{array}\right), \quad \bar{X}^{M}=\left(\begin{array}{c}
\partial_{-} \bar{\theta}^{\alpha} \\
\bar{\Pi}^{\mu} \\
\bar{d}_{\alpha} \\
\frac{1}{2} \bar{N}^{\mu \nu}
\end{array}\right), \\
{[M=(\alpha, \mu, \alpha, \mu \nu)],}
\end{gathered}
$$

of which the components are defined as

$$
\begin{aligned}
\Pi^{\mu} & =\partial_{+} x^{\mu}+\frac{1}{2} \theta^{\alpha}\left(\Gamma^{\mu}\right)_{\alpha \beta} \partial_{+} \theta^{\beta}, \\
\bar{\Pi}^{\mu} & =\partial_{-} x^{\mu}+\frac{1}{2} \bar{\theta}^{\alpha}\left(\Gamma^{\mu}\right)_{\alpha \beta} \partial_{-} \bar{\theta}^{\beta}, \\
d_{\alpha} & =\pi_{\alpha}-\frac{1}{2}\left(\Gamma_{\mu} \theta\right)_{\alpha}\left[\partial_{+} x^{\mu}+\frac{1}{4}\left(\theta \Gamma_{\mu} \partial_{+} \theta\right)\right], \\
\bar{d}_{\alpha} & =\bar{\pi}_{\alpha}-\frac{1}{2}\left(\Gamma_{\mu} \bar{\theta}\right)_{\alpha}\left[\partial_{-} x^{\mu}+\frac{1}{4}\left(\bar{\theta} \Gamma_{\mu} \partial_{-} \bar{\theta}\right)\right], \\
N^{\mu \nu} & =\frac{1}{2} w_{\alpha}\left(\Gamma^{[\mu \nu]}\right)^{\alpha}{ }_{\beta} \lambda^{\beta}, \quad \bar{N}^{\mu \nu}=\frac{1}{2} \bar{w}_{\alpha}\left(\Gamma^{[\mu \nu]}\right)^{\alpha}{ }_{\beta} \bar{\lambda}^{\beta} .
\end{aligned}
$$

In the analysis we will use the action in the form (2.1). Just for completeness, the expanded form of the action is

$$
\begin{aligned}
S= & \int \mathrm{d}^{2} \xi\left[\partial_{+} \theta^{\alpha} A_{\alpha \beta} \partial_{-} \bar{\theta}^{\beta}+\partial_{+} \theta^{\alpha} A_{\alpha \mu} \bar{\Pi}^{\mu}\right. \\
& +\Pi^{\mu} A_{\mu \alpha} \partial_{-} \bar{\theta}^{\alpha}+\Pi^{\mu} A_{\mu \nu} \bar{\Pi}^{v}+d_{\alpha} E^{\alpha}{ }_{\beta} \partial_{-} \bar{\theta}^{\beta} \\
& +d_{\alpha} E^{\alpha}{ }_{\mu} \bar{\Pi}^{\mu}+\partial_{+} \theta^{\alpha} E_{\alpha} \beta \bar{d}_{\beta}+\Pi^{\mu} \bar{E}_{\mu}{ }^{\beta} \bar{d}_{\beta}+d_{\alpha} \mathrm{P}^{\alpha \beta} \bar{d}_{\beta} \\
& +\frac{1}{2} N^{\mu v} \Omega_{\mu \nu, \beta} \partial_{-} \bar{\theta}^{\beta}+\frac{1}{2} N^{\mu v} \Omega_{\mu \nu, \rho} \bar{\Pi}^{\rho}
\end{aligned}
$$




$$
\begin{aligned}
& +\frac{1}{2} \partial_{+} \theta^{\alpha} \Omega_{\alpha, \mu \nu} \bar{N}^{\mu \nu}+\frac{1}{2} \Pi^{\mu} \Omega_{\mu, \nu \rho} \bar{N}^{\nu \rho}+\frac{1}{2} N^{\mu \nu} \bar{C}_{\mu \nu}{ }^{\beta} \bar{d}_{\beta} \\
& \left.+\frac{1}{2} d_{\alpha} C^{\alpha}{ }_{\mu \nu} \bar{N}^{\mu \nu}+\frac{1}{4} N^{\mu \nu} S_{\mu \nu, \rho \sigma} \bar{N}^{\rho \sigma}\right]+S_{\lambda}+S_{\bar{\lambda}},
\end{aligned}
$$

where we used matrix $A_{M N}$ in explicit form

$$
A_{M N}=\left(\begin{array}{cccc}
A_{\alpha \beta} & A_{\alpha \nu} & E_{\alpha} \beta & \Omega_{\alpha, \mu \nu} \\
A_{\mu \beta} & A_{\mu \nu} & \bar{E}_{\mu} \beta & \Omega_{\mu, \nu \rho} \\
E_{\beta}^{\alpha} & E^{\alpha}{ }_{\nu} & \mathrm{P}^{\alpha \beta} & C^{\alpha}{ }_{\mu \nu} \\
\Omega_{\mu \nu, \beta} & \Omega_{\mu \nu, \rho} & \bar{C}_{\mu \nu} \beta & S_{\mu \nu, \rho \sigma}
\end{array}\right) .
$$

The matrix $A_{M N}$, containing type II superfields, generally depends on $x^{\mu}, \theta^{\alpha}$ and $\bar{\theta}^{\alpha}$. The superfields $A_{\mu \nu}, \bar{E}_{\mu}{ }^{\alpha}, E^{\alpha}{ }_{\mu}$ and $\mathrm{P}^{\alpha \beta}$ are physical superfields, because their first components are supergravity fields. The fields in the first column and first row are auxiliary superfields because they can be expressed in terms of the physical ones [18]. The remaining ones, $\Omega_{\alpha, \mu \nu}, \Omega_{\mu \nu, \alpha}, \Omega_{\mu, \nu \rho}\left(\Omega_{\mu \nu, \rho}\right), C^{\alpha}{ }_{\mu \nu}\left(\bar{C}_{\mu \nu}{ }^{\alpha}\right)$ and $S_{\mu \nu, \rho \sigma}$, are curvatures (field strengths).

The world sheet $\Sigma$ is parameterized by $\xi^{m}=\left(\xi^{0}=\right.$ $\left.\tau, \xi^{1}=\sigma\right)$ and $\partial_{ \pm}=\partial_{\tau} \pm \partial_{\sigma}$. The superspace is spanned by bosonic coordinates $x^{\mu}(\mu=0,1,2, \ldots, 9)$ and fermionic ones $\theta^{\alpha}$ and $\bar{\theta}^{\alpha}(\alpha=1,2, \ldots, 16)$. The variables $\pi_{\alpha}$ and $\bar{\pi}_{\alpha}$ are the momenta canonically conjugated to $\theta^{\alpha}$ and $\bar{\theta}^{\alpha}$, respectively. The actions for the pure spinors, $S_{\lambda}$ and $S_{\bar{\lambda}}$, are free field actions,

$S_{\lambda}=\int \mathrm{d}^{2} \xi w_{\alpha} \partial_{-} \lambda^{\alpha}, \quad S_{\bar{\lambda}}=\int \mathrm{d}^{2} \xi \bar{w}_{\alpha} \partial_{+} \bar{\lambda}^{\alpha}$,

where $\lambda^{\alpha}$ and $\bar{\lambda}^{\alpha}$ are pure spinors and $w_{\alpha}$ and $\bar{w}_{\alpha}$ are their canonically conjugated momenta, respectively. The pure spinors satisfy the so-called pure spinor constraints,

$\lambda^{\alpha}\left(\Gamma^{\mu}\right)_{\alpha \beta} \lambda^{\beta}=\bar{\lambda}^{\alpha}\left(\Gamma^{\mu}\right)_{\alpha \beta} \bar{\lambda}^{\beta}=0$

We are going to perform T-dualization along some subset of the bosonic coordinates $x^{a}$. So, we will assume that these directions are Killing vectors and that the background fields do not depend on them.

\section{T-dualization along arbitrary number of coordinates}

In this section we will make T-dualization along an arbitrary subset of the coordinates $x^{a}$. First we will make a mathematical preparation extracting the desired directions from variables $X^{M}$ and $\bar{X}^{M}$. Then we will apply a standard Buscher procedure assuming that the background fields do not depend on $x^{a}$.

\subsection{Mathematical preparation}

In order to make T-dualization along arbitrary bosonic directions $x^{a}$, let us split the spacetime index $\mu$ into $a$ and the undualized ones, $i$. We write the variables $X^{M}$ and $\bar{X}^{N}$ in the appropriate form, separating derivatives of T-dualized coordinates $x^{a}$,

$X^{M}=P^{M}{ }_{a} \partial_{+} x^{a}+{ }_{a} j_{+}^{M} \equiv P^{M}{ }_{a} \partial_{+} x^{a}+P^{M}{ }_{i} \partial_{+} x^{i}+j_{+}^{M}$,
$\bar{X}^{M}=\bar{P}^{M}{ }_{a} \partial_{-} x^{a}+{ }_{a} j_{-}^{M} \equiv \bar{P}^{M}{ }_{a} \partial_{-} x^{a}+\bar{P}^{M}{ }_{i} \partial_{-} x^{i}+j_{-}^{M}$,

where

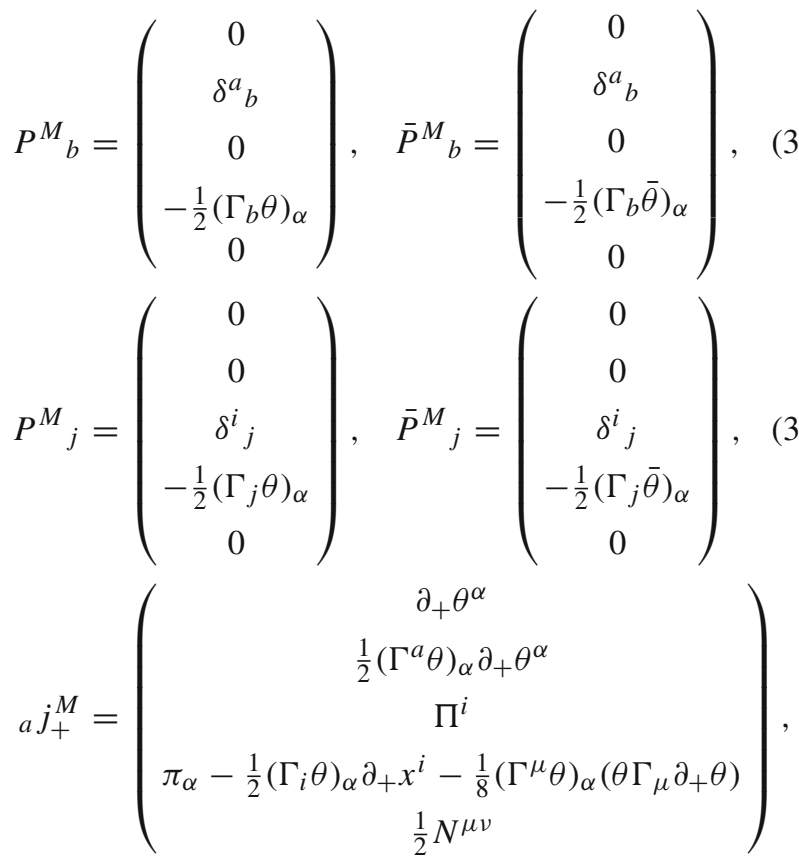

$a j_{-}^{M}=\left(\begin{array}{c}\partial_{-} \bar{\theta}^{\alpha} \\ \frac{1}{2}\left(\Gamma^{a} \bar{\theta}\right)_{\alpha} \partial_{-} \bar{\theta}^{\alpha} \\ \bar{\Pi}^{i} \\ \bar{\pi}_{\alpha}-\frac{1}{2}\left(\Gamma_{i} \bar{\theta}\right)_{\alpha} \partial_{-} x^{i}-\frac{1}{8}\left(\Gamma^{\mu} \bar{\theta}\right)_{\alpha}\left(\bar{\theta} \Gamma_{\mu} \partial_{-} \bar{\theta}\right) \\ \frac{1}{2} \bar{N}^{\mu \nu}\end{array}\right)$.

In comparison with (2.2) we split $\Pi^{\mu}$ into $\Pi^{a}$ and $\Pi^{i}$ and $\Gamma^{\mu}$ into $\Gamma^{a}$ and $\Gamma^{i}$. Consequently, the variables $X^{M}$ and $\bar{X}^{M}$ have five block components and $A_{M N}$ is a $5 \times 5$ block matrix, where the index $M=(\alpha, a, i, \alpha, \mu \nu)$.

Let us introduce the notation

$$
\begin{aligned}
\tilde{\mathcal{A}}_{a b}= & P_{a}{ }^{M} A_{M N} \bar{P}^{N}{ }_{b}=A_{a b}-\frac{1}{2} \bar{E}_{a}{ }^{\alpha}\left(\Gamma_{b} \bar{\theta}\right)_{\alpha} \\
& -\frac{1}{2}\left(\Gamma_{a} \theta\right)_{\alpha} E^{\alpha}{ }_{b}+\frac{1}{4}\left(\Gamma_{a} \theta\right)_{\alpha} P^{\alpha \beta}\left(\Gamma_{b} \bar{\theta}\right)_{\beta},
\end{aligned}
$$




$$
\begin{aligned}
\tilde{\Pi}_{+a b} & \equiv \frac{1}{\kappa} \tilde{\mathcal{A}}_{a b}, \quad \tilde{\Pi}_{ \pm a b}=\tilde{B}_{a b} \pm \frac{1}{2} \tilde{G}_{a b}, \\
J_{+a} & =\frac{2}{\kappa}{ }_{a} j_{+}^{M} A_{M N} \bar{P}_{a}^{N}, \quad J_{-a}=-\frac{2}{\kappa} P_{a}{ }^{M} A_{M N a} j_{-}^{N} .
\end{aligned}
$$

The field $\tilde{B}_{a b}$ plays the role of a Kalb-Ramond field and $\tilde{G}_{a b}$ the role of the metric in the process of T-dualization.

Applying this decomposition to the action (2.1), it gets the form

$$
\begin{aligned}
S= & \int \mathrm{d}^{2} \xi\left(\kappa \partial_{+} x^{a} \tilde{\Pi}_{+a b} \partial_{-} x^{b}-\frac{\kappa}{2} \partial_{+} x^{a} J_{-a}\right. \\
& \left.+\frac{\kappa}{2} J_{+a} \partial_{-} x^{a}+{ }_{a} j_{+}^{M} A_{M N} a j_{-}^{N}\right),
\end{aligned}
$$

where all terms with derivatives $\partial_{ \pm} x^{a}$ are written explicitly.

\subsection{Buscher procedure}

Let us perform T-dualization of the action (3.9) along the $x^{a}$ directions. We assume that the $x^{a}$ directions are Killing ones; therefore, background fields do not depend on them. Applying the standard procedure of Buscher T-dualization we replace ordinary world-sheet derivatives $\partial_{ \pm} x^{a}$ by covariant ones,

$D_{ \pm} x^{a}=\partial_{ \pm} x^{a}+v_{ \pm}^{a}$.

In order to make the fields $v_{ \pm}^{a}$ unphysical we add the term

$S_{L}=\frac{\kappa}{2} \int \mathrm{d}^{2} \xi\left(v_{+}^{a} \partial_{-} y_{a}-v_{-}^{a} \partial_{+} y_{a}\right)$,

where the $y_{a}$ are Lagrange multipliers. Taking into account that $x^{a}$ are isometry directions we choose the gauge $x^{a}=0$, so that the gauge fixed action takes the form

$$
\begin{aligned}
S_{f i x}= & \int \mathrm{d}^{2} \xi\left(\kappa v_{+}^{a} \tilde{\Pi}_{+a b} v_{-}^{b}-\frac{\kappa}{2} v_{+}^{a} J_{-a}+\frac{\kappa}{2} J_{+a} v_{-}^{a}\right. \\
& \left.+{ }_{a} j_{+}^{M} A_{M N a} j_{-}^{N}\right)+\frac{\kappa}{2} \int \mathrm{d}^{2} \xi\left(v_{+}^{a} \partial_{-} y_{a}-v_{-}^{a} \partial_{+} y_{a}\right) .
\end{aligned}
$$

By the equations of motion for $y_{a}$ we find that the field strength is equal to zero,

$\partial_{+} v_{-}^{a}-\partial_{-} v_{+}^{a}=0$

its solution is $v_{ \pm}^{a}=\partial_{ \pm} x^{a}$. In this way the action $S_{f i x}$ turns to the initial action $S$.

On the equations of motion for gauge fields $v_{ \pm}^{a}$ we have

$$
\begin{aligned}
& \partial_{+} y_{a}=2 v_{+}^{b} \tilde{\Pi}_{+b a}+J_{+a}, \\
& \partial_{-} y_{a}=-2 \tilde{\Pi}_{+a b} v_{-}^{b}+J_{-a} .
\end{aligned}
$$

Substituting the expressions for $v_{ \pm}^{a}$, $v_{+}^{a}=\frac{1}{2}\left(\partial_{+} y_{b}-J_{+b}\right)\left(\tilde{\Pi}_{+}^{-1}\right)^{b a}$,

$v_{-}^{a}=-\frac{1}{2}\left(\tilde{\Pi}_{+}^{-1}\right)^{a b}\left(\partial_{-} y_{b}-J_{-b}\right)$,

into $S_{f i x}$ we get the T-dual action

$$
\begin{aligned}
{ }_{a} S= & \int \mathrm{d}^{2} \xi\left[\frac{\kappa}{4} \partial_{+} y_{a}\left(\tilde{\Pi}_{+}^{-1}\right)^{a b} \partial_{-} y_{b}\right. \\
& +\frac{1}{2} \partial_{+} y_{a}\left(\tilde{\Pi}_{+}^{-1}\right)^{a b} A_{b N a} j_{-}^{N}-\frac{1}{2} a^{M} j_{+}^{M} \bar{A}_{M a}\left(\tilde{\Pi}_{+}^{-1}\right)^{a b} \partial_{-} y_{b} \\
& \left.+a j_{+}^{M}\left(A_{M N}-\frac{1}{\kappa} \bar{A}_{M a}\left(\tilde{\Pi}_{+}^{-1}\right)^{a b} A_{b N}\right) a j_{-}^{N}\right]
\end{aligned}
$$

where we used the expressions for the currents (3.4) and (3.5) and introduced the definitions

$\bar{A}_{M a} \equiv A_{M N} \bar{P}_{a}^{N}, \quad A_{a M} \equiv P_{a}{ }^{N} A_{N M}$.

\subsection{T-dual transformation laws}

In order to obtain the relation between the initial coordinates $x^{a}$ and the corresponding T-dual ones $y_{a}$, we eliminate $v_{ \pm}$ from the equations of motion for the Lagrange multipliers $y_{a}, v_{ \pm}^{a}=\partial_{ \pm} x^{a}$, and the other ones for the gauge fields $v_{ \pm}^{a}$ (3.14) and (3.15),

$\partial_{ \pm} y_{a} \cong-2 \tilde{\Pi}_{\mp a b} \partial_{ \pm} x^{b}+J_{ \pm a}$.

Using the expressions for the currents $a j_{ \pm}^{M}=j_{ \pm}^{M}+$ $P^{M}{ }_{i} \partial_{ \pm} x^{i}$ given in Eq. (3.1), we get the currents (3.8) in the form

$J_{ \pm a}=\bar{J}_{ \pm a}-2 \tilde{\Pi}_{\mp a i} \partial_{ \pm} x^{i}$,

where we introduced the notation

$$
\begin{gathered}
\bar{J}_{+a}=\frac{2}{\kappa} j_{+}^{M} A_{M N} \bar{P}_{a}^{N}, \quad \bar{J}_{-a}=-\frac{2}{\kappa} P_{a}{ }^{M} A_{M N} j_{-}^{N}, \\
\tilde{\Pi}_{+i a} \equiv \frac{1}{\kappa} P_{i}{ }^{M} A_{M N} \bar{P}_{a}^{N}, \quad \tilde{\Pi}_{+a i}=\frac{1}{\kappa} P_{a}{ }^{M} A_{M N} \bar{P}^{N}{ }_{i} .
\end{gathered}
$$

Now we can rewrite the transformation law (3.20) in the form

$\partial_{ \pm} y_{a} \cong-2 \tilde{\Pi}_{\mp a b} \partial_{ \pm} x^{b}-2 \tilde{\Pi}_{\mp a i} \partial_{ \pm} x^{i}+\bar{J}_{ \pm a}$,

while the inverse one is

$\partial_{ \pm} x^{a} \cong-2 \kappa \tilde{\theta}_{ \pm}^{a b} \tilde{\Pi}_{\mp b i} \partial_{ \pm} x^{i}-\kappa \tilde{\theta}_{ \pm}^{a b}\left(\partial_{ \pm} y_{b}-\bar{J}_{ \pm b}\right)$.

Here we introduced the field $\tilde{\theta}_{ \pm}^{a b}$ :

$\tilde{\theta}_{ \pm}^{a b}=-\frac{2}{\kappa}\left(\hat{g}^{-1} \tilde{\Pi}_{ \pm} \tilde{G}^{-1}\right)^{a b}, \quad \hat{g}_{a b}=\left(\tilde{G}-4 \tilde{B} \tilde{G}^{-1} \tilde{B}\right)_{a b}$,

such that

$\tilde{\theta}_{ \pm}^{a b} \tilde{\Pi}_{\mp b c}=\frac{1}{2 \kappa} \delta^{a}{ }_{c}$. 
Note that the form of the transformation laws is the same as in the case of constant background fields [13]. But now all background fields depend on the undualized coordinates $\left(\theta^{\alpha}, \bar{\theta}^{\alpha}, x^{i}\right)$.

Let us find the relation between the complete T-dual coordinates ${ }_{a} X_{\hat{\mu}}=\left\{y_{a}, x^{i}\right\}$ and the initial ones $x^{\mu}$. Together with T-dual transformation laws (3.24), which relate $y_{a}$ with $x^{\mu}$, we can simply add $\partial_{ \pm} x^{i}=\partial_{ \pm} x^{i}$ and rewrite both relations in the form

$$
\begin{aligned}
\partial_{+}\left({ }_{a} X\right)_{\hat{\mu}} & =\left(\bar{Q}^{-1 T}\right)_{\hat{\mu} \nu} \partial_{+} x^{\nu}+\bar{J}_{+\hat{\mu}}, \\
\partial_{-}\left({ }_{a} X\right)_{\hat{\mu}} & =\left(Q^{-1 T}\right)_{\hat{\mu} \nu} \partial_{-} x^{\nu}+\bar{J}_{-\hat{\mu}} .
\end{aligned}
$$

The matrices

$$
\begin{aligned}
Q^{\hat{\mu} v} & =\left(\begin{array}{cc}
\kappa \tilde{\theta}_{+}^{a b} & 0 \\
-2 \kappa \tilde{\Pi}_{-i c} \tilde{\theta}_{+}^{c b} & \delta_{j}^{i}
\end{array}\right), \\
\bar{Q}^{\hat{\mu} v} & =\left(\begin{array}{cc}
\kappa \tilde{\theta}_{-}^{a b} & 0 \\
-2 \kappa \tilde{\Pi}_{+i c} \tilde{\theta}_{-}^{c b} & \delta_{j}^{i}
\end{array}\right),
\end{aligned}
$$

and theirs inverses

$Q_{\mu \hat{v}}^{-1}=\left(\begin{array}{cc}2 \tilde{\Pi}_{-a b} & 0 \\ 2 \tilde{\Pi}_{-i b} & \delta_{i}^{j}\end{array}\right), \quad \bar{Q}_{\mu \hat{v}}^{-1}=\left(\begin{array}{cc}2 \tilde{\Pi}_{+a b} & 0 \\ 2 \tilde{\Pi}_{+i b} & \delta_{i}^{j}\end{array}\right)$,

perform T-dualization for the vector indices. The currents are defined as

$\bar{J}_{ \pm \hat{\mu}}=\left(\begin{array}{c}\bar{J}_{ \pm a} \\ 0\end{array}\right)$.

\subsection{Two sets of vielbeins and gamma matrices}

Different chiralities transform differently as in Refs. [13,15, 16]. Consequently, there are two types of T-dual vielbeins, defined by

${ }_{a} e^{\underline{a} \hat{\mu}}=e^{\underline{a}}{ }_{\nu}\left(Q^{T}\right)^{v \hat{\mu}}, \quad{ }_{a} e^{\underline{a} \hat{\mu}}=e^{\underline{a}}{ }_{\nu}\left(\bar{Q}^{T}\right)^{v \hat{\mu}}$,

producing the same T-dual metric ${ }_{a} G^{\hat{\mu} \hat{v}}$, where hat indices are from the T-dual picture. Two types of vielbeins produce two sets of gamma matrices in the T-dual picture,

${ }_{a} \Gamma_{\hat{\mu}}=\left({ }_{a} e^{-1}\right)_{\hat{\mu} \underline{a}} \Gamma^{\underline{a}}=\left({ }_{a} e^{-1} \Gamma\right) \hat{\mu}$,

${ }_{a} \bar{\Gamma}_{\hat{\mu}}=\left({ }_{a} \bar{e}^{-1}\right)_{\hat{\mu} \underline{a}} \Gamma^{\underline{a}}=\left({ }_{a} \bar{e}^{-1} \Gamma\right)_{\hat{\mu}}$,

which are connected by the local Lorentz transformation

${ }_{a} \bar{\Gamma}_{\hat{\mu}}={ }_{a} \Omega^{-1}{ }_{a} \Gamma_{\hat{\mu}}{ }_{a} \Omega$.

Here ${ }_{a} \Omega$ is the spinorial representation of the Lorentz transformation

${ }_{a} \Omega^{-1} \Gamma^{\underline{a}}{ }_{a} \Omega=\left(\Lambda^{-1}\right)^{\underline{a}} \underline{b} \Gamma^{\underline{b}}$.
The underlined indices are Lorentz ones (denoted by $\underline{a}, \underline{b}$ ). The matrix $\Lambda^{\underline{a}}{ }_{b}$ is a matrix of the Lorentz transformation and it is given by the expression

$\Lambda^{\underline{a}} \underline{b}=e^{\underline{a}} \mu\left(Q^{-1} \bar{Q}\right)^{T \mu}{ }_{\nu}\left(e^{-1}\right)^{v}{ }_{\underline{b}}$.

In T-dual theory, as a consequence of two types of $\Gamma$ matrices, there are two types of T-dual supersymmetry invariant variables:

${ }_{a} d_{\alpha}={ }_{a} \pi_{\alpha}-\frac{1}{2}\left({ }_{a} \Gamma^{\hat{\mu}}{ }_{a} \theta\right)_{\alpha}\left(\partial_{+}{ }_{a} X_{\hat{\mu}}+\frac{1}{4}{ }_{a} \theta_{a} \Gamma_{\hat{\mu}} \partial_{+}{ }_{a} \theta\right)$,

${ }_{a} \bar{d}_{\alpha}={ }_{a} \bar{\pi}_{\alpha}-\frac{1}{2}\left({ }_{a} \bar{\Gamma}^{\hat{\mu}}{ }_{a} \bar{\theta}\right)_{\alpha}\left(\partial_{-}{ }_{a} X_{\hat{\mu}}+\frac{1}{4}{ }_{a} \bar{\theta}_{a} \bar{\Gamma}_{\hat{\mu}} \partial_{-}{ }_{a} \bar{\theta}\right)$.

In order to work with one set of gamma $\Gamma$ matrices we have to introduce proper variables. We can rewrite the bar expression as

$$
\begin{aligned}
\left({ }_{a} \Omega_{a} \bar{d}\right)_{\alpha}= & \left({ }_{a} \Omega_{a} \bar{\pi}\right)_{\alpha}-\frac{1}{2}\left({ }_{a} \Gamma^{\hat{\mu}}{ }_{a} \Omega_{a} \bar{\theta}\right)_{\alpha} \\
& \times\left(\partial_{-} X_{\hat{\mu}}+\frac{1}{4}{ }_{a} \bar{\theta}{ }_{a} \Omega^{-1}{ }_{a} \Gamma_{\hat{\mu}}{ }_{a} \Omega \partial_{-a} \bar{\theta}\right) .
\end{aligned}
$$

Let us preserve the expressions for the unbar variables, ${ }_{a} \theta^{\alpha}=$ $\theta^{\alpha}$ and $\pi_{\alpha}=\pi_{\alpha}$, and change the bar variables,

$\bullet^{-} \bar{\theta}^{\alpha} \equiv{ }_{a} \Omega^{\alpha}{ }_{\beta} \bar{\theta}^{\beta}, \quad \cdot \bar{\pi}_{\alpha} \equiv{ }_{a} \Omega_{\alpha}{ }^{\beta}{ }_{a} \bar{\pi}_{\beta}$.

Now the forms of the transformation of the supersymmetric invariants are the same. In short, the fermionic index without bar is unchanged, while the bar fermionic index is multiplied by ${ }_{a} \Omega$.

The further story, finding the spinorial representation of the local Lorentz symmetry $a$ connecting the two kinds of vielbeins, is the same as in $[13,15,16]$ and we will not repeat it. We will just write the final expression for the matrix ${ }_{a} \Omega$ in the spinorial representation,

$$
\begin{aligned}
{ }_{a} \Omega & =\sqrt{\prod_{i=1}^{d} G_{a_{i} a_{i}}{ }_{a} \Gamma\left(i \Gamma^{11}\right)^{d}} \\
\Gamma^{11} & =(i)^{\frac{D(D-1)}{2}} \frac{1}{\prod_{\mu=0}^{D-1} G_{\mu \mu}} \varepsilon_{\mu_{1} \mu_{2} \ldots \mu_{D}} \Gamma^{\mu_{1}} \Gamma^{\mu_{2}} \ldots \Gamma^{\mu_{D}} .
\end{aligned}
$$

The matrix $\Gamma^{11}$ has a normalization constant to satisfy the condition $\left(\Gamma^{11}\right)^{2}=1$. Also we have

$$
{ }_{a} \Gamma=(i)^{\frac{d(d-1)}{2}} \prod_{i=1}^{d} \Gamma^{a_{i}}=(i)^{\frac{d(d-1)}{2}} \Gamma^{a_{1}} \Gamma^{a_{2}} \cdots \Gamma^{a_{d}},
$$


so that

$\left({ }_{a} \Gamma\right)^{2}=\prod_{i=1}^{d} G^{a_{i} a_{i}}=\frac{1}{\prod_{i=1}^{d} G_{a_{i} a_{i}}}$.

This matrix is introduced in analogy to $\Gamma^{11}$ in the subspace spanned by the T-dualized directions $x^{a}$. The symbol $d$ denotes the number of T-dualized coordinates $x^{a} \quad(a=$ $1,2, \ldots, d)$.

\section{Relations between initial and T-dual background fields}

In this section we will find the most general form of the Tdual background fields in terms of the initial ones in the type II pure spinor superstring under simultaneous T-dualization. We will also discuss the form of the T-dual dilaton field obtained in the quantization procedure.

We expect that the T-dual action (3.18) has the form of the initial action (2.1) but expressed in terms of the T-dual variables and background fields,

${ }_{a} S=\int \mathrm{d}^{2} \xi{ }_{a} X_{\hat{M}^{a}}^{T} A^{\hat{M} \hat{N}}{ }_{a} \bar{X}_{\hat{N}}$,

where in analogy with (3.1) we have

$$
\begin{aligned}
& { }_{a} X_{\hat{M}}={ }_{a} \hat{P}_{\hat{M}}^{a} \partial_{+} y_{a}+{ }_{a}^{\star} j_{+\hat{M}}, \\
& { }_{a} \bar{X}_{\hat{M}}={ }_{a} \hat{\bar{P}}_{\hat{M}}{ }^{a} \partial_{-} y_{a}+{ }_{a}^{\star} \bar{j}_{-\hat{M}}, \quad[\hat{M}=(\alpha, \hat{\mu}, \alpha, \hat{\mu} \hat{\nu})] .
\end{aligned}
$$

Let us recall that the index $\hat{\mu}$ means that the index of the T-dualized direction, $a$, goes up if it was down in the initial theory and vice versa, while the index $i$ keeps the position.

The decomposition of the T-dual variables ${ }_{a} X_{\hat{M}}$ and ${ }_{a} \bar{X}_{\hat{M}}$ has a similar form to the initial ones, $X^{M}$ and $\bar{X}^{M}$. We express the T-dual currents ${ }_{a}^{\star} j_{+} \hat{M}^{\text {and }}{ }_{a}^{\star} \bar{j}_{-} \hat{M}$ in terms of the initial ones $a j_{ \pm}^{M}$ as

$\stackrel{\star}{a} j_{+\hat{M}}=\omega_{\hat{M} N} a j_{+}^{N}, \quad \stackrel{\star}{a} \bar{j}_{-\hat{M}}=\bar{\omega}_{\hat{M} N} a j_{-}^{N}$,

in order to make a comparison of the actions (4.1) and (3.18), which will produce the relations between T-dual and initial background fields. We did not write free field actions for the pure spinors, $S_{\lambda}$ and $S_{\bar{\lambda}}$, because they carry fermionic indices while we T-dualize along some subset of bosonic indices. Thus, they are not changed in the process of T-dualization.

Following the form of the initial theory we introduced for the T-dual case, we have

$$
{ }_{a} \hat{P}_{\hat{M}}{ }^{a}=\left(\begin{array}{c}
0 \\
\delta_{a}^{b} \\
0 \\
\frac{\kappa}{2} \tilde{\theta}_{-}^{a b}\left(\Gamma_{b} \theta\right)_{\alpha} \\
0,
\end{array}\right),{ }_{a} \hat{\bar{P}}_{\hat{M}}{ }^{a}=\left(\begin{array}{c}
0 \\
\delta_{a}^{b} \\
0 \\
\frac{\kappa}{2} \tilde{\theta}_{+}^{a b}\left(\Gamma_{b}^{\bullet} \bar{\theta}_{\beta} \Omega^{\beta}{ }_{\alpha}\right. \\
0
\end{array}\right) .
$$

The matrices $\omega$ and $\bar{\omega}$ are of the form

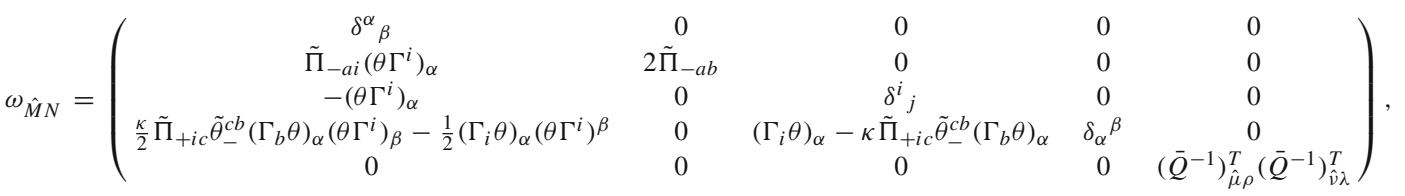

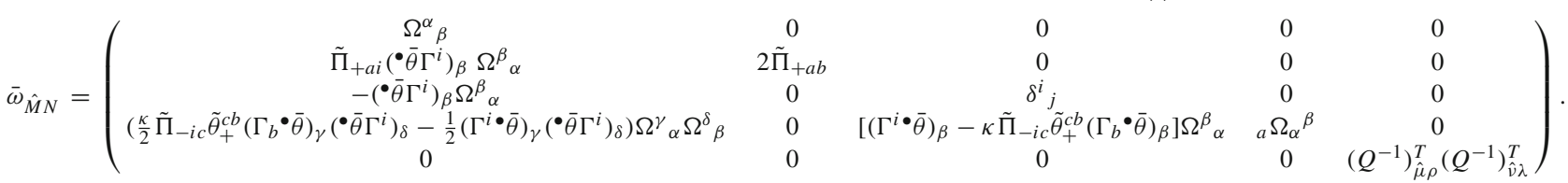

We also will need the inverse matrices, $\omega^{-1}$ and $\bar{\omega}^{-1}$,

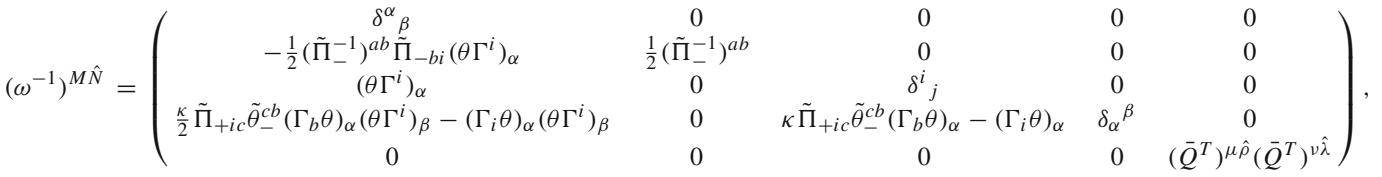

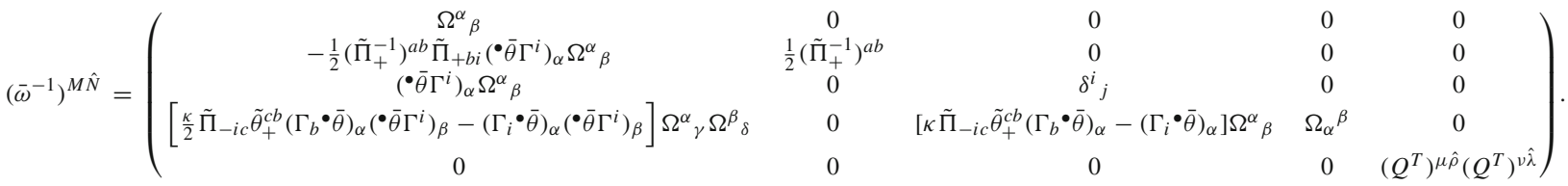


During the calculation of the above matrices we used the expressions for the T-dual gamma matrices with upper and lower indices,

${ }_{a} \Gamma_{\hat{\mu}}=-\left(Q^{-1}\right)_{\hat{\mu} \nu}^{T} \Gamma^{\nu}=\left(\begin{array}{c}a \Gamma_{a} \\ a \Gamma^{i}\end{array}\right)=\left(\begin{array}{c}2 \tilde{\Pi}_{+a b} \Gamma^{b}+2 \tilde{\Pi}_{+a i} \Gamma^{i} \\ -\Gamma^{i}\end{array}\right)$,

${ }_{a} \Gamma^{\hat{\mu}}=-Q^{\hat{\mu} v} \Gamma_{\nu}=\left(\begin{array}{c}a \Gamma^{a} \\ { }_{a} \Gamma_{i}\end{array}\right)=\left(\begin{array}{c}-\kappa \tilde{\theta}_{+}^{a b} \Gamma_{b} \\ -\Gamma_{i}+2 \kappa \tilde{\Pi}_{-i c} \tilde{\theta}_{+}^{c b} \Gamma_{b}\end{array}\right)$,

${ }_{a} \bar{\Gamma}_{\hat{\mu}}=-\left(\bar{Q}^{-1}\right)_{\hat{\mu} \nu}^{T} \Gamma^{\nu}=\left(\begin{array}{c}a \bar{\Gamma}_{a} \\ \bar{\Gamma}^{i}\end{array}\right)=\left(\begin{array}{c}2 \tilde{\Pi}_{-a b} \Gamma^{b}+2 \tilde{\Pi}_{-a i} \Gamma^{i} \\ -\Gamma^{i}\end{array}\right)$,

${ }_{a} \bar{\Gamma}^{\hat{\mu}}=-\bar{Q}^{\hat{\mu} v} \Gamma_{\nu}=\left(\begin{array}{c}a \bar{\Gamma}^{a} \\ a \bar{\Gamma}_{i}\end{array}\right)=\left(\begin{array}{c}-\kappa \tilde{\theta}_{-}^{a b} \Gamma_{b} \\ -\Gamma_{i}+2 \kappa \tilde{\Pi}_{+i c} \tilde{\theta}_{-}^{c b} \Gamma_{b}\end{array}\right)$.

The explicit form of the action given in (4.1) is

$$
\begin{aligned}
{ }_{a} S= & \int \mathrm{d}^{2} \xi\left(\partial_{+} y_{a} \hat{P}^{T a}{ }_{\hat{M} a} A^{\hat{M}} \hat{N} \hat{\bar{P}}_{\hat{N}}^{b} \partial_{-} y_{b}\right. \\
& +\partial_{+} y_{a} \hat{P}^{T a}{ }_{\hat{M} a} A^{\hat{M} \hat{N}} \bar{\omega}_{\hat{N} P} a j_{-}^{P} \\
& +{ }_{a} j_{+}^{N} \omega_{N \hat{M}{ }^{a} A^{\hat{M}} \hat{N}} \hat{\bar{P}}_{\hat{N}} b \partial_{-} y_{b} \\
& \left.+{ }_{a} j_{+}^{M} \omega_{M \hat{P}}^{T}{ }^{T} A^{\hat{P}} \hat{Q}_{\bar{\omega}} \hat{Q}_{N} a j_{-}^{N}\right) .
\end{aligned}
$$

Comparing this action with that from Eq. (3.18) we obtain the T-dual fields in terms of the initial ones,

$$
\begin{aligned}
& \hat{P}^{T a}{ }_{\hat{M} a} A^{\hat{M} \hat{N}} \hat{\bar{P}}_{\hat{N}}^{b}=\frac{\kappa}{4}\left(\tilde{\Pi}_{+}^{-1}\right)^{a b} \Longrightarrow{ }_{a} \tilde{\Pi}_{+}^{a b}=\frac{1}{4}\left(\tilde{\Pi}_{+}^{-1}\right)^{a b}, \\
& { }_{a} A^{a \hat{M}}=\frac{1}{2}\left(\tilde{\Pi}_{+}^{-1}\right)^{a b} A_{b P}\left(\bar{\omega}^{-1}\right)^{P \hat{M}}, \\
& { }_{a} \bar{A}^{\hat{M} a}=-\frac{1}{2}\left(\omega^{T}\right)^{-1 \hat{M} N} \bar{A}_{N b}\left(\tilde{\Pi}_{+}^{-1}\right)^{b a}, \\
& { }_{a} A^{\hat{M} \hat{N}}=\left(\omega^{T}\right)^{-1 \hat{M} P}\left(A_{P Q}-\frac{1}{\kappa} \bar{A}_{P a}\left(\tilde{\Pi}_{+}^{-1}\right)^{a b} A_{b Q}\right)\left(\bar{\omega}^{-1}\right)^{Q \hat{N}},
\end{aligned}
$$

where

$$
{ }_{a} A^{a \hat{N}} \equiv \hat{P}^{T a}{ }_{\hat{M} a} A^{\hat{M} \hat{N}}, \quad{ }_{a} \bar{A}^{\hat{M} a} \equiv{ }_{a} A^{\hat{M} \hat{N}} \hat{\bar{P}}_{\hat{N}}{ }^{a} .
$$

The next step is to express the components of the T-dual fields in terms of the components of the initial background fields. Also in order to find the transformation law for the physical superfield components in $A_{M N}$ we need the explicit expressions

$$
{ }_{a} A^{a \hat{N}}=\left(\begin{array}{c}
{ }_{a} A^{a}{ }_{\alpha}+\frac{\kappa}{2} \tilde{\theta}_{a}^{a b}\left(\Gamma_{b} \theta\right)_{\beta}{ }_{a} E^{\beta}{ }_{\alpha} \\
{ }_{a} A^{a b}+\frac{\kappa}{2} \tilde{\theta}_{a}^{a b}\left(\Gamma_{b} \theta\right)_{\beta} E^{\beta b} \\
{ }_{a} A^{a}{ }_{i}+\frac{\kappa}{2} \tilde{\theta}_{-}^{a b}\left(\Gamma_{b} \theta\right)_{\beta} E^{\beta}{ }_{i} \\
{ }_{a} \bar{E}^{a \beta}+\frac{\kappa}{2} \tilde{\theta}_{-}^{a b}\left(\Gamma_{b} \theta\right)_{\alpha} P^{\alpha \beta} \\
{ }_{a} \Omega^{a, \hat{\mu} \hat{v}}+\frac{\kappa}{2} \tilde{\theta}_{-}^{a b}\left(\Gamma_{b} \theta\right)_{\alpha} C^{\alpha, \hat{\mu} \hat{\nu}}
\end{array}\right)^{T},
$$

$$
\begin{aligned}
& { }_{a} \bar{A}^{\hat{M} a}=\left(\begin{array}{c}
{ }_{a} A_{\alpha}{ }^{a}+\frac{\kappa}{2} a E_{\alpha} \beta \tilde{\theta}_{+}^{a b}\left(\Gamma_{b} \bullet \bar{\theta}\right)_{\gamma} \Omega_{\beta}{ }_{\beta} \\
{ }_{a} A^{a b}+\frac{\kappa}{2} a \bar{E}^{a \beta} \tilde{\theta}_{+}^{b c}\left(\Gamma_{c} \cdot \bar{\theta}\right)_{\gamma} \Omega_{\beta}^{\gamma} \\
{ }_{a} A_{i}{ }^{a}+\frac{\kappa}{2} a \bar{E}_{i} \beta \tilde{\theta}_{+}^{a b}\left(\Gamma_{b} \bullet \bar{\theta}\right)_{\gamma} \Omega_{\beta}^{\gamma} \\
{ }_{a} E^{\alpha a}+\frac{\kappa}{2} a P^{\alpha \beta} \tilde{\theta}_{+}^{a b}\left(\Gamma_{b} \bullet \bar{\theta}\right)_{\gamma} \Omega_{\beta}^{\gamma} \\
{ }_{a} \Omega^{\hat{\mu} \hat{v}, a}+\frac{\kappa}{2} a \bar{C}^{\hat{\mu} \hat{v}, \beta} \tilde{\theta}_{+}^{a b}\left(\Gamma_{b} \bullet \bar{\theta}\right)_{\gamma} \Omega_{\beta}^{\gamma}
\end{array}\right), \\
& A_{a M}=\left(\begin{array}{c}
A_{a \alpha}-\frac{1}{2}\left(\Gamma_{a} \theta\right)_{\beta} E^{\beta}{ }_{\alpha} \\
A_{a b}-\frac{1}{2}\left(\Gamma_{a} \theta\right)_{\alpha} E^{\alpha}{ }_{b} \\
A_{a i}-\frac{1}{2}\left(\Gamma_{a} \theta\right)_{\alpha} E^{\alpha}{ }_{i} \\
\bar{E}_{a}{ }^{\alpha}-\frac{1}{2}\left(\Gamma_{a} \theta\right)_{\beta} P^{\beta \alpha} \\
\Omega_{a, \mu \nu}-\frac{1}{2}\left(\Gamma_{a} \theta\right)_{\alpha} C^{\alpha}{ }_{\mu \nu}
\end{array}\right)^{T}, \\
& \bar{A}_{M a}=\left(\begin{array}{c}
A_{\alpha a}-\frac{1}{2} E_{\alpha} \beta\left(\Gamma_{a} \bar{\theta}\right)_{\beta} \\
A_{a b}-\frac{1}{2} \bar{E}_{a}^{\beta}\left(\Gamma_{b} \bar{\theta}\right)_{\beta} \\
A_{i a}-\frac{1}{2} \bar{E}_{i} \beta\left(\Gamma_{a} \bar{\theta}\right)_{\beta} \\
E^{\alpha}{ }_{a}-\frac{1}{2} P^{\alpha \beta}\left(\Gamma_{a} \bar{\theta}\right)_{\beta} \\
\Omega_{\mu \nu, a}-\frac{1}{2} \bar{C}_{\mu \nu}{ }^{\beta}\left(\Gamma_{a} \bar{\theta}\right)_{\beta}
\end{array}\right) .
\end{aligned}
$$

In order to describe the dilaton field $\Phi$ in the standard formulation one should add a Fradkin-Tseytlin term, as in [16]

$S_{\Phi}=\int \mathrm{d}^{2} \xi \sqrt{-g} R^{(2)} \Phi$,

to the initial action. Here $R^{(2)}$ is the scalar curvature of the world sheet. It is well known that the dilaton field transformation under T-dualization is considered within the path integral formalism [13-16,20,21]. For a constant background the Gaussian path integral produces the expression $\left(\operatorname{det} \tilde{\Pi}_{+a b}\right)^{-1}$.

In this article we T-dualize just along a subset of coordinates $x^{a}$ and assume that all background fields are independent of $x^{a}$. Consequently, gaussian integration over gauge fields $v_{ \pm}^{a}$ in a path integral produces the same result as in the so-called constant background case [13]; thus we get the form of the T-dual dilaton field,

${ }_{a} \Phi\left(x^{i}, \theta^{\alpha}, \bar{\theta}^{\alpha}\right)=\Phi\left(x^{i}, \theta^{\alpha}, \bar{\theta}^{\alpha}\right)-\ln \operatorname{det}\left(2 \tilde{\Pi}_{+a b}\right)$.

Using the expression for $\tilde{\Pi}_{+a b}$ (3.7) in the form

$\tilde{\Pi}_{+a b}=\Pi_{+a b}-\Delta_{a b}$,

where $\Delta_{a b}$ is defined as

$$
\begin{aligned}
\Delta_{a b}= & \frac{1}{2 \kappa} \bar{E}_{a}^{\alpha}\left(\Gamma_{b} \bar{\theta}\right)_{\alpha}+\frac{1}{2 \kappa}\left(\Gamma_{a} \theta\right)_{\alpha} E_{b}^{\alpha} \\
& -\frac{1}{4 \kappa}\left(\Gamma_{a} \theta\right)_{\alpha} P^{\alpha \beta}\left(\Gamma_{b} \bar{\theta}\right)_{\beta},
\end{aligned}
$$

we get

$$
\begin{aligned}
{ }_{a} \Phi\left(x^{i}, \theta^{\alpha}, \bar{\theta}^{\alpha}\right)= & \Phi\left(x^{i}, \theta^{\alpha}, \bar{\theta}^{\alpha}\right)-\ln \operatorname{det}\left(2 \Pi_{+a c}\right) \\
& -\ln \operatorname{det}\left(\mathbb{1}-\Pi_{+}^{-1} \Delta\right)^{c}{ }_{b} .
\end{aligned}
$$

The quantity $\Delta_{a b}$ measures the difference between general case considered in this article and the case considered in 
Ref. [13]. Note that the expressions for the background fields in Ref. [13] have been obtained from the general ones in Ref. [18] after elimination of all $\theta^{\alpha}$ and $\bar{\theta}^{\alpha}$ dependent terms in the action. In that manner in Ref. [13] we omitted all terms in $\Delta_{a b}$. From Eq. (4.26) we see that ignoring $\theta^{\alpha}$ and $\bar{\theta}^{\alpha}$ dependent terms in [13] prevents us from getting a complete solution for the T-dual dilaton.

\section{The components of the T-dual matrix ${ }_{a} A^{\hat{M} \hat{N}}$}

In this section we will write explicit expressions for the Tdual superfields and, considering that the background fields are constant, compare the results with the already known constant background case.

\subsection{The physical superfields and comparison with constant background case}

In order to find the T-dual field ${ }_{a} A^{a b}$ we take into consideration some particular component of Eqs. (4.15)-(4.17).

The second component of Eq. (4.15) $(\hat{M} \rightarrow b)$. It produces the equation

$$
\begin{aligned}
& { }_{a} A^{a b}+\frac{\kappa}{2} \tilde{\theta}_{-}^{a b}\left(\Gamma_{b} \theta\right)_{\alpha a} E^{\alpha b} \\
& \quad=\frac{1}{2}\left(\tilde{\Pi}_{+}^{-1}\right)^{a c}\left[A_{c d}-\frac{1}{2}\left(\Gamma_{c} \theta\right)^{\alpha} E_{d}^{\alpha}\right] \cdot \frac{1}{2}\left(\tilde{\Pi}_{+}^{-1}\right)^{d b} .
\end{aligned}
$$

We treat the left-hand side and the right-hand side of this equation as an expansion in powers of $\theta^{\alpha}$. Equating appropriate coefficients we obtain the T-dual fields ${ }_{a} A^{a b}$ and ${ }_{a} E^{\alpha a}$,

${ }_{a} A^{a b}=\frac{1}{4}\left(\tilde{\Pi}_{+}^{-1}\right)^{a c} A_{c d}\left(\tilde{\Pi}_{+}^{-1}\right)^{d b}$,

${ }_{a} E^{\alpha a}=\frac{1}{2}\left(\tilde{\Pi}_{-}^{-1}\right)^{a b} E_{b}{ }^{\alpha} . \quad\left(\tilde{\Pi}_{-}=-\Pi_{+}^{T}\right)$.

Using the redefinitions $A_{a b}=\kappa \Pi_{+a b}$ and ${ }_{a} A^{a b}=\kappa_{a} \Pi_{+}^{a b}$ and the relation $\tilde{\theta}_{-}^{a b} \tilde{\Pi}_{+b c}=\frac{1}{2 \kappa} \delta^{a}{ }_{c}$, we get

${ }_{a} \Pi_{+}^{a b}=\frac{1}{4}\left(\tilde{\Pi}_{+}^{-1}\right)^{a c} \Pi_{+c d}\left(\tilde{\Pi}_{+}^{-1}\right)^{d b}$,

${ }_{a} E^{\alpha a}=\kappa \tilde{\theta}_{+}^{a b} E^{\alpha}{ }_{b}$.

In the case of constant background fields $\left(\Delta_{a b}=0\right)$, Eq. (5.3) transforms into

${ }_{a} \Pi_{+}^{a b}=\frac{1}{4}\left(\Pi_{+}^{-1}\right)^{a b}=\frac{\kappa}{2} \hat{\theta}_{-}^{a b}$,

because in that case $\tilde{\Pi}_{+a b}=\Pi_{+a b}$ and $\tilde{\theta}_{ \pm}^{a b} \rightarrow \hat{\theta}_{ \pm}^{a b}$.

Equation (5.4) in the limit of constant background fields is in accordance with the appropriate result obtained in [13]. Here we have in mind that the field $\Psi_{\mu}^{\alpha}$ is a zeroth order term in the expansion of $E_{\mu}^{\alpha}$ [18], which produces

${ }_{a} \Psi^{\alpha a}=\kappa \hat{\theta}_{+}^{a b} \Psi_{b}^{\alpha}$.
The second component of Eq. (4.16) $(\hat{M} \rightarrow a)$. It produces

$$
\begin{aligned}
& { }_{a} A^{a b}+\frac{\kappa}{2}{ }_{a} \bar{E}^{a \beta} \tilde{\theta}_{+}^{b c}\left(\Gamma_{c}^{\bullet} \bar{\theta}\right)_{\gamma} \Omega_{\beta}^{\gamma} \\
& =-\frac{1}{2}\left(-\frac{1}{2}\right)\left(\tilde{\Pi}_{+}^{-1}\right)^{a c}\left[A_{c d}-\frac{1}{2} \bar{E}_{c}^{\beta}\left(\Gamma_{d} \bar{\theta}\right)_{\beta}\right]\left(\tilde{\Pi}_{+}^{-1}\right)^{d b},
\end{aligned}
$$

and we again get the expression for ${ }_{a} A^{a b}$, but we additionally have

${ }_{a} \bar{E}^{a \alpha}=\kappa \tilde{\theta}_{-}^{a b} \bar{E}_{b}^{\beta} \Omega_{\beta}^{\alpha}$

In the constant background case $\bar{E}_{a}{ }^{\alpha} \rightarrow \bar{\Psi}_{a}{ }^{\alpha}$. Consequently, here we also have the correct constant background limit,

${ }_{a} \bar{\Psi}^{\alpha a}=\kappa \hat{\theta}_{-}^{a b} \bar{\Psi}_{b}^{\beta} \Omega_{\beta}^{\alpha}$.

It is useful to observe the fact that the expressions for the T-dual fields ${ }_{a} \bar{E}^{a \alpha}$ and ${ }_{a} E^{a \alpha}$ can be obtained analyzing the fourth components of Eqs. (4.15) and (4.16), respectively. Also note that $\hat{\theta}_{ \pm}^{a b}$ appearing in (5.6) and (5.9) is a constant tensor, defined as the inverse of the constant tensor $\Pi_{+a b}[13]$.

The third component of Eq. (4.15) $(\hat{M} \rightarrow i)$. Let us consider the equation which follows from the third component of Eq. (4.15) $(\hat{M} \rightarrow i)$,

$$
\begin{aligned}
{ }_{a} A^{a}{ }_{i} & +\frac{\kappa}{2} \tilde{\theta}_{-}^{a b}\left(\Gamma_{b} \theta\right)_{\beta a} E^{\beta}{ }_{i} \\
= & \frac{1}{2}\left(\tilde{\Pi}_{+}^{-1}\right)^{a b}\left(A_{b j}-\frac{1}{2}\left(\Gamma_{b} \theta\right)_{\beta} E^{\beta}{ }_{i}\right) \\
& +\frac{1}{2}\left(\tilde{\Pi}_{+}^{-1}\right)^{a b}\left(\bar{E}_{b}^{\alpha}-\frac{1}{2}\left(\Gamma_{b} \theta\right)_{\beta} P^{\beta \alpha}\right) \\
& \times\left(\kappa \tilde{\Pi}_{-i c} \tilde{\theta}_{+}^{c b}\left(\Gamma_{b} \bullet \bar{\theta}\right)_{\gamma}-\left(\Gamma_{i}^{\bullet} \bar{\theta}\right)_{\gamma}\right) \Omega_{\alpha}^{\gamma} .
\end{aligned}
$$

Extracting the zero components in the expansion we get

${ }_{a} A^{a}{ }_{i}=\frac{1}{2}\left(\tilde{\Pi}_{+}^{-1}\right)^{a b} A_{b i}$.

In order to make the comparison with the constant background case easier, we introduce the following notation:

${ }_{a} A^{a}{ }_{i}=\kappa_{a} \Pi_{+i}^{a}, \quad A_{a i}=\kappa \Pi_{+a i}$,

and, using Eq. (3.27), we obtain

${ }_{a} \Pi_{+i}^{a}=\kappa \tilde{\theta}_{-}^{a b} \Pi_{+b i}$.

Treating the third component of Eq. (4.16) $(\hat{M} \rightarrow i)$,

$$
\begin{array}{r}
{ }_{a} A_{i}{ }^{a}+\frac{\kappa}{2}{ }_{a} \bar{E}_{i}{ }^{\alpha} \tilde{\theta}_{+}^{a b}\left(\Gamma_{b} \bullet \bar{\theta}\right)_{\beta} \Omega^{\beta}{ }_{\alpha} \\
=-\frac{1}{2}\left(A_{i b}-\frac{1}{2} \bar{E}_{i}{ }^{\beta}\left(\Gamma_{b} \bar{\theta}\right)_{\beta}\right)
\end{array}
$$




$$
\begin{aligned}
& -\frac{1}{2}\left(\kappa \tilde{\Pi}_{+i c} \tilde{\theta}_{-}^{c b}\left(\Gamma_{b} \theta\right)_{\alpha}-\left(\Gamma_{i} \theta\right)_{\alpha}\right) \\
& \times\left(E_{d}^{\alpha}-\frac{1}{2} P^{\alpha \beta}\left(\Gamma_{d} \bar{\theta}\right)_{\beta}\right)\left(\tilde{\Pi}_{+}^{-1}\right)^{d a},
\end{aligned}
$$

in the same way as in the previous case, we have

$$
{ }_{a} \Pi_{+i}{ }^{a}=-\kappa \Pi_{+i b} \tilde{\theta}_{-}^{b a} .
$$

The last two expressions, (5.13) and (5.15), in the limit of the constant background fields are in full correspondence with the result obtained in the constant background case [13].

The $(\hat{M} \rightarrow i, \hat{N} \rightarrow j)$ component of Eq. (4.17). Considering the appropriate component in Eq. (4.17) $(\hat{M} \rightarrow i, \hat{N} \rightarrow$ $j$ ), we obtain

$$
\begin{aligned}
{ }_{a} A_{i j}= & A_{i j}-\frac{1}{\kappa}\left[A_{i a}-\frac{1}{2} \bar{E}_{i}^{\alpha}\left(\Gamma_{a} \bar{\theta}\right)_{\alpha}\right]\left(\tilde{\Pi}_{+}^{-1}\right)^{a b} \\
& \times\left[A_{b j}-\frac{1}{2}\left(\Gamma_{b} \theta\right)_{\delta} E^{\delta}{ }_{j}\right] .
\end{aligned}
$$

Using the redefinitions, $A_{i j}=\kappa \Pi_{+i j}$ and ${ }_{a} A_{i j}=\kappa_{a} \Pi_{+i j}$, this relation can be rewritten in the form

$$
\begin{aligned}
{ }_{a} \Pi_{+i j}= & \Pi_{+i j}-\frac{1}{\kappa^{2}}\left[\kappa \Pi_{+i a}-\frac{1}{2} \bar{E}_{i}^{\alpha}\left(\Gamma_{a} \bar{\theta}\right)_{\alpha}\right]\left(\tilde{\Pi}_{+}^{-1}\right)^{a b} \\
& \times\left[\kappa \Pi_{+b j}-\frac{1}{2}\left(\Gamma_{b} \theta\right)_{\delta} E_{j}^{\delta}\right] .
\end{aligned}
$$

In the constant background case explicit $\theta^{\alpha}$ and $\bar{\theta}^{\alpha}$ dependence disappears and $\tilde{\Pi}_{+a b}=\Pi_{+a b}$. Consequently, we get

${ }_{a} \Pi_{+i j}=\Pi_{+i j}-2 \kappa \Pi_{+i a} \hat{\theta}_{-}^{a b} \Pi_{+b j}$,

which is exactly the relation obtained in the constant background case [13].

Equation (4.17) where $(\hat{M} \rightarrow i, \hat{N} \rightarrow \alpha),(\hat{M} \rightarrow$ $\alpha, \hat{N} \rightarrow i)$ and $(\hat{M} \rightarrow \alpha, \hat{N} \rightarrow \beta)$. Also we read in this case, respectively,

$$
\begin{aligned}
{ }_{a} \bar{E}_{i}{ }^{\alpha}= & \left\{E_{i}{ }^{\gamma}-\frac{1}{\kappa}\left[A_{i a}-\frac{1}{2} \bar{E}_{i} \beta\left(\Gamma_{a} \bar{\theta}\right)_{\beta}\right]\left(\tilde{\Pi}_{+}^{-1}\right)^{a b}\right. \\
& \left.\times\left[\bar{E}_{b}{ }^{\alpha}-\frac{1}{2}\left(\Gamma_{b} \theta\right)_{\delta} P^{\delta \gamma}\right]\right\} \Omega_{\gamma}{ }^{\alpha}, \\
{ }_{a} E^{\alpha}{ }_{i}= & E^{\alpha}{ }_{i}-\frac{1}{\kappa}\left[E^{\alpha}{ }_{a}-\frac{1}{2} P^{\alpha \beta}\left(\Gamma_{a} \bar{\theta}\right)_{\beta}\right]\left(\tilde{\Pi}_{+}^{-1}\right)^{a b} \\
& \times\left[A_{b i}-\frac{1}{2}\left(\Gamma_{b} \theta\right)_{\gamma} E^{\gamma}{ }_{i}\right], \\
{ }_{a} P^{\alpha \beta}= & \left\{P^{\alpha \gamma}-\frac{1}{\kappa}\left[E^{\alpha}{ }_{a}-\frac{1}{2} P^{\alpha \delta}\left(\Gamma_{a} \bar{\theta}\right)_{\delta}\right]\left(\tilde{\Pi}_{+}^{-1}\right)^{a b}\right. \\
& \left.\times\left[\bar{E}_{b}{ }^{\gamma}-\frac{1}{2}\left(\Gamma_{b} \theta\right)_{\epsilon} P^{\epsilon \gamma}\right]\right\} \Omega_{\gamma}{ }^{\beta} .
\end{aligned}
$$

In the constant background limit, which effectively means that we put $\theta^{\alpha}=\bar{\theta}^{\alpha}=0, A_{i a}=\kappa \Pi_{+i a}, A_{b i}=\kappa \Pi_{+b i}$,
$\left(\tilde{\Pi}_{+}^{-1}\right)^{a b}=2 \kappa \hat{\theta}_{-}^{a b}$ and $P^{\alpha \beta}=\frac{1}{2 \kappa} e^{\frac{\Phi}{2}} F^{\alpha \beta}$, we obtain the relations from [13],

$$
\begin{aligned}
{ }_{a} \bar{\Psi}_{i}^{\alpha} & =\left[\bar{\Psi}_{i}{ }^{\beta}-2 \kappa \Pi_{+i a} \hat{\theta}_{-}^{a b} \bar{\Psi}_{b}{ }^{\beta}\right] \Omega_{\beta}{ }^{\alpha}, \\
{ }_{a} \Psi^{\alpha}{ }_{i} & =\Psi^{\alpha}{ }_{i}-2 \kappa \Psi^{\alpha}{ }_{a} \hat{\theta}_{-}^{a b} \Pi_{+b i}, \\
e^{\frac{a \Phi}{2}}{ }_{a} F^{\alpha \beta} & =\left[e^{\frac{\Phi}{2}} F^{\alpha \gamma}-4 \kappa \Psi^{\alpha}{ }_{a} \hat{\theta}_{-}^{a b} \bar{\Psi}_{b}{ }^{\gamma}\right] \Omega_{\gamma}{ }^{\beta} .
\end{aligned}
$$

Our compact result of the general case (4.15)-(4.17) in components has the form (5.1), (5.7), (5.10), (5.14), (5.16) and (5.19)-(5.21). It gives the right limit for the constant background fields (5.5), (5.6), (5.9), (5.13), (5.15), (5.18) and (5.22)-(5.24).

\subsection{T-dual auxiliary background fields and field strengths}

The part of the main result beside the full expressions for the T-dual physical superfields are the expressions for the T-dual auxiliary superfields (the first column and the first row in matrix ${ }_{a} A^{\hat{M} \hat{N}}$ ) and expressions for the T-dual field strengths (the last column and the last row in matrix ${ }_{a} A^{\hat{M}} \hat{N}$ ). These background fields are absent in the already considered constant background case [13], because the imposed assumptions eliminated them from the theory (a detailed argumentation is in [13] and [18]).

In order to read all mentioned background fields we use the appropriate components of (4.15)-(4.17). Fixing the indices $\hat{M}$ and $\hat{N}$, we get the equations which we treat, as in the previous cases, as expansions in powers of $\theta^{\alpha}$ and $\bar{\theta}^{\alpha}$. Equating the appropriate coefficients in the expansions, we read the form of the T-dual background fields. Because there are many expressions and some of them are long, we write just a few of them. For example, we give the form of the background field T-dual to the $A_{a \alpha}$ and the field strength T-dual to the $\Omega_{a, \mu \nu}$. Using Eqs. (4.15) and (4.19)-(4.22), after straightforward calculation, we get

$$
\begin{aligned}
(\hat{M} \rightarrow \alpha) \quad{ }_{a} A^{a}{ }_{\alpha} & =\kappa \tilde{\theta}_{-}^{a b} A_{b \beta}{ }_{a} \Omega^{\beta}{ }_{\alpha}, \\
(\hat{M} \rightarrow \hat{\mu} \hat{\nu}) \quad{ }_{a} \Omega^{a, \hat{\mu} \hat{v}} & =\kappa \tilde{\theta}_{-}^{a b} \Omega_{b, \rho \lambda}\left(Q^{T}\right)^{\rho \hat{\mu}}\left(Q^{T}\right)^{\lambda \hat{v}},
\end{aligned}
$$

while considering the component of Eq. (4.17) where $\hat{M} \rightarrow$ $\hat{\mu} \hat{\nu}$ and $\hat{N} \rightarrow \hat{\lambda} \hat{\rho}$, we obtain

$$
\begin{aligned}
{ }_{a} S^{\hat{\mu} \hat{v}, \hat{\lambda} \hat{\rho}}= & \bar{Q}^{\hat{\mu} \mu} \bar{Q}^{\hat{v} v}\left[S_{\mu \nu, \lambda \rho}-\left(\Omega_{\mu \nu, a}-\frac{1}{2} \bar{C}_{\mu \nu}{ }^{\alpha}\left(\Gamma_{a} \bar{\theta}\right)_{\alpha}\right)\right. \\
& \left.\cdot \tilde{\theta}_{-}^{a b}\left(\Omega_{b, \lambda \rho}-\frac{1}{2}\left(\Gamma_{b} \theta\right)_{\beta} C^{\beta}{ }_{\lambda \rho}\right)\right]\left(Q^{T}\right)^{\lambda \hat{\lambda}}\left(Q^{T}\right)^{\rho \hat{\rho}} .
\end{aligned}
$$

\section{Concluding remarks}

In this paper we have investigated simultaneous T-dualization of the pure spinor type II superstring described by the action 
of Ref. [18]. We assumed that the background fields do not depend on the coordinates along which we make the T-dualization. Our goal was to find the form of the Tdual background fields, especially T-dual auxiliary fields and field strengths which are not present in the constant background case. To be compared to the articles $[15,16]$, where single direction T-dualization is performed, here we demonstrated simultaneous T-dualization along some subset, $x^{a}(a=1,2, \ldots d)$, of the spacetime directions. Also following Refs. $[13,15,16]$, we found the form of the spinorial representation of the local Lorentz transformation ${ }_{a} \Omega$ occurring in the T-dual picture.

The action we used in this article is type II superstring action in the pure spinor formulation of Ref. [18]. It is derived using nilpotency and (anti)holomorphicity conditions as an expansion in powers of $\theta^{\alpha}$ and $\bar{\theta}^{\alpha}$. In Ref. [13] we considered a constant background version of this action obtained under certain assumptions - the background fields are independent of all $x^{\mu}$ coordinates and we take just the first components in the expansions of the background fields. In this way we lost information as regards the form of the T-dual auxiliary background fields and field strengths, and the complete form of the T-dual physical superfields.

It is difficult to work with the expanded form of the action (2.6), because it has a large number of terms. We used a condensed form of the action (2.1) and extracted in the variables $X^{M}$ and $\bar{X}^{M}$ terms containing derivatives of the directions along which we T-dualize, $\partial_{ \pm} x^{a}$. The remaining part of these variables is denoted as the current $a j_{ \pm}^{M}$. We inserted that expression into the action and made T-dualization along the $x^{a}$ direction. On the equation of motion for gauge fields $v_{ \pm}^{a}$ we obtained the T-dual action expressed in terms of T-dual coordinates $y_{a}$ and currents ${ }_{a} j_{ \pm}^{M}$. Under T-dualization the form of the action is preserved and consequently, expressing the T-dual action in terms of the T-dual variables and fields, we finally got all T-dual background fields in the considered general case. In order to compare them with the constant background case of Ref. [13] we explicitly wrote the expressions for the physical superfields. In the limit of the constant background fields, the obtained expressions turn into the expressions of Ref. [13].

Combining the equations of motion for the Lagrange multipliers $y_{a}$ and for the gauge fields $v_{ \pm}^{a}$ we obtain the T-dual transformation laws (3.28) in the most general case of a type II pure spinor superstring. Let us stress that we consider the general case and that all background fields now depend on the undualized directions $x^{i}, \theta^{\alpha}$ and $\bar{\theta}^{\alpha}$. Because the two chiral sectors transform differently, there are two sets of vielbeins and gamma matrices. We obtain the general form of the local Lorentz transformation in the spinorial representation ${ }_{a} \Omega$ connecting the two chiral sectors. In order to work with properly defined variables and background fields, fermions with bar index are multiplied by the matrix ${ }_{a} \Omega$.
The T-dual transformation of the dilaton field $\Phi\left(x^{i}, \theta^{\alpha}\right.$, $\left.\bar{\theta}^{\alpha}\right)$ is treated within the quantum formalism. In this paper, using the matrices $\tilde{\Pi}_{+a b}$ and $\tilde{\Pi}_{+}^{a b}$, we obtained the general expression for the T-dual dilaton field (4.24).

Consequently, in this article we performed the Buscher simultaneous T-dualization of type II superstring in a pure spinor formulation and found the general form of the T-dual transformation laws and the full expressions for the T-dual background fields.

Open Access This article is distributed under the terms of the Creative Commons Attribution 4.0 International License (http://creativecomm ons.org/licenses/by/4.0/), which permits unrestricted use, distribution, and reproduction in any medium, provided you give appropriate credit to the original author(s) and the source, provide a link to the Creative Commons license, and indicate if changes were made. Funded by $\mathrm{SCOAP}^{3}$.

\section{References}

1. E. Witten, Nucl. Phys. B 443, 85 (1995)

2. T. Banks, W. Fischler, S.H. Shenker, L. Susskind, Phys. Rev. D 55, 5112 (1997)

3. M.J. Duff, Int. J. Mod. Phys. A 11, 5623-5642 (1996)

4. M.J. Duff, J.T. Liu, R. Minasian, Nucl. Phys. B 452, 261-282 (1995)

5. M.J. Duff, P.S. Howe, T. Inami, K.S. Stelle, Phys. Lett. B 191, 70 (1985)

6. P. Horava, E. Witten, Nucl. Phys. B 460, 506-524 (1996)

7. C.M. Hull, P.K. Townsend, Nucl. Phys. B 438, 109-137 (1995)

8. C.M. Hull, R.R. Khuri, Nucl. Phys. B 536, 219-244 (1999)

9. R. Dijkgraaf, E. Verlinde, H. Verlinde, Nucl. Phys. B 500, 43-61 (1997)

10. A. Kehagias, Phys. Lett. B 377, 241-244 (1996)

11. M. Cvetic, H. Lu, C.N. Pope, K.S. Stelle, Nucl. Phys. B 573, 149 176 (2000)

12. I. Jeon, K. Lee, J.-H. Park, Y. Suh, Phys. Lett. B 723, 245-250 (2013)

13. B. Nikolic, B. Sazdovic, Eur. Phys. J. C 77, 197 (2017)

14. A. Giveon, M. Roček, Nucl. Phys. B 421, 173 (1994)

15. S.F. Hassan, Nucl. Phys. B 568, 145 (2000)

16. R. Benichou, G. Policastro, J. Troost, Phys. Lett. B 661, 129 (2008)

17. E. Plauschinn, Nucl. Phys. B 893, 257-286 (2015)

18. P.A. Grassi, L. Tamassia, JHEP 07, 071 (2004)

19. C.M. Hull, JHEP 07, 021 (1998)

20. T. Buscher, Phys. Lett. B 194, 59 (1987)

21. T. Buscher, Phys. Lett. 201, 466 (1988)

22. J. Polchinski, String Theory, vol. II (Cambridge University Press, Cambridge, 1998)

23. B. Zwiebach, A First Course in String Theory (Cambridge University Press, Cambridge, 2004)

24. K. Becker, M. Becker, J. Schwarz, String Theory and M-Theory: A Modern Introduction (Cambridge University Press, Cambridge, 2007)

25. M. Roček, E. Verlinde, Nucl. Phys. B 373, 630 (1992)

26. A. Giveon, M. Porrati, E. Rabinovici, Phys. Rep. 244, 77 (1994)

27. E. Alvarez, L. Alvarez-Gaume, J. Barbon, Y. Lozano, Nucl. Phys. B 415, 71 (1994)

28. B. Sazdović, Eur. Phys. J. C 77, 634 (2017)

29. F. Cordonier-Tello, D. Lust, E. Plauschinn, JHEP 08, 198 (2018) 
30. N. Berkovits, arXiv:hep-th/0209059

31. P.A. Grassi, G. Policastro, P. van Nieuwenhuizen, JHEP 10, 054 (2002)

32. P.A. Grassi, G. Policastro, P. van Nieuwenhuizen, JHEP 11, 004 (2002)

33. P.A. Grassi, G. Policastro, P. van Nieuwenhuizen, Adv. Theor. Math. Phys. 7, 499 (2003)

34. P.A. Grassi, G. Policastro, P. van Nieuwenhuizen, Phys. Lett. B 553, $96(2003)$
35. N. Berkovits, JHEP 07, 091 (2015)

36. N. Berkovits, H. Gomez, arXiv:1711.09966

37. M. Cederwall, Springer Proc. Phys. 153, 61-93 (2014)

38. J. de Boer, P.A. Grassi, P. van Nieuwenhuizen, Phys. Lett. B 574, 98 (2003)

39. N. Berkovits, P. Howe, Nucl. Phys. B 635, 75 (2002) 